\title{
Possible topological contribution to the anomalous Hall effect of the noncollinear ferromagnet $\mathrm{Fe}_{3} \mathrm{Sn}_{2}$
}

\author{
Christopher D. O'Neill, ${ }^{1, *}$ Andrew S. Wills, ${ }^{2,3}$ and Andrew D. Huxley ${ }^{1}$ \\ ${ }^{1}$ School of Physics and Astronomy and CSEC, University of Edinburgh, Edinburgh EH9 3JZ, United Kingdom \\ ${ }^{2}$ Chemistry Department, UCL, 20 Gordon Street, London WC1H OAJ, United Kingdom \\ ${ }^{3}$ London Centre for Nanotechnology, 17-19 Gordon Street, London WC1H OAH, United Kingdom
}

(Received 8 May 2019; revised manuscript received 1 August 2019; published 18 November 2019)

\begin{abstract}
The magnetization, magnetoresistance, and Hall effect of the kagome structured material $\mathrm{Fe}_{3} \mathrm{Sn}_{2}$ is reported for high-quality single crystals. Previous investigations of $\mathrm{Fe}_{3} \mathrm{Sn}_{2}$ polycrystals detected ferromagnetism at $T_{c} \approx$ $657 \mathrm{~K}$ with moments along the easy $c$ axis and a moment rotation towards the $a-b$ plane upon cooling that culminates in moments freezing to form a spin glass at $T_{F} \approx 80 \mathrm{~K}$. The results presented here for single crystals show the lower value of $T_{F}=70 \pm 2.5 \mathrm{~K}$, most likely due to the increased sample quality. Above $T_{F}$ we identify a topological contribution to the Hall resistivity coinciding with an anomalous magnetoresistance. This supports recent proposals that the magnetic structure contains magnetic skyrmions in this temperature range.
\end{abstract}

DOI: 10.1103/PhysRevB.100.174420

\section{INTRODUCTION}

Topologically protected quantum phenomena are known to exist in a wide range of condensed matter systems. One such state with promising use in technological applications is the topological Hall effect (THE) in materials with magnetic spin structures that exhibit chirality [1]. It is realizable for materials containing whirls of magnetic spins known as skyrmions such as $\mathrm{MnSi}$ [2] and other complex nontrivial spin structures like those of geometrically frustrated pyrochlores [3]. Electrons passing through the chiral structure acquire a Berry phase contribution that leads to a topological Hall effect in addition to a background from the ordinary Lorentz force Hall effect and anomalous Hall effect (AHE) present in magnetic materials. For large enough effects the THE contribution may be separated from the background effects in transport experiments, for example, in $\mathrm{MnSi}[4,5]$.

Here we discuss the Hall effect in metallic $\mathrm{Fe}_{3} \mathrm{Sn}_{2}$, which has a kagome crystal structure well known in the context of geometrically frustrated magnetic spins. In $\mathrm{Fe}_{3} \mathrm{Sn}_{2}$, ferromagnetism with noncollinear spins indicative of frustration has been reported [6], while in an applied field the presence of skyrmion like spin-textured bubbles has been detected $[7,8]$. This makes $\mathrm{Fe}_{3} \mathrm{Sn}_{2}$ an interesting candidate in which to look for a THE. We report Hall effect, magnetization, and magnetoresistance measurements on high-quality single crystals of $\mathrm{Fe}_{3} \mathrm{Sn}_{2}$. An extra component of the Hall resistivity, $\Delta \rho_{x y}$, can be isolated after subtraction of the ordinary and anomalous Hall effect contributions that bears a striking resemblance to a THE. The existence of $\Delta \rho_{x y}$ is complimented by an anomaly in magnetoresistance over the same temperature and field range.

$\mathrm{Fe}_{3} \mathrm{Sn}_{2}$ has the space group $R \overline{3} m$ and $\mathrm{Fe}$ atoms form kagome planes made up of two sizes of equilateral triangles,

*chris.oneill@ed.ac.uk of side length 2.732 and $2.582 \AA$, shown in red and blue, respectively, in Fig. 1(a). The kagome planes form offset bilayers where the smaller $\mathrm{Fe}$-Fe distances make up octahedra, shown in Fig. 1(b). The Sn atoms sit within the kagome layers and between the bilayers $[6,9,10]$. Previous reports using Mössbauer spectroscopy [11-13] and magnetometry $[6,14]$ detected ferromagnetism with moments along the $c$ axis below the Curie temperature, $T_{C}\left(T_{C}=657-612 \mathrm{~K}\right.$, depending on the sample). Upon cooling below $250 \mathrm{~K}$, a continuous rotation of the moments from the $c$ axis into the $a-b$ plane was detected with Mössbauer spectroscopy $[12,13]$. However, the magnetic susceptibility, $M / H$, indicates that the rotation begins at a much higher temperature [6]. A change in slope of $M / H$ below $\approx 520 \mathrm{~K}$ resembles the onset of a small-angle moment rotation and upon further cooling a sharper change of slope at $\approx 300 \mathrm{~K}$ suggests an abrupt increase in rotation angle for temperatures below this. Powder neutron diffraction patterns reveal that the moment rotation in $\mathrm{Fe}_{3} \mathrm{Sn}_{2}$ is noncollinear, consistent with magnetic frustration due to a strong competition between the magnetic interactions present [6]. The competition between magnetic interactions is evidenced further by a second transition, reminiscent of moments freezing to a spin-glass state, at $T_{F} \approx 80 \mathrm{~K}$ [6]. The exact details of the spin-glass state are unknown.

Pereiro et al. [15] showed that competing magnetic interactions in a kagome lattice can result in skyrmion spin structured excitations. Experimentally, it appears that in $\mathrm{Fe}_{3} \mathrm{Sn}_{2}$ such skyrmions may be static. The evidence comes from Lorentz transmission electron microscopy in an applied magnetic field, $\mu_{0} H$, parallel $(\|)$ to the $c$ axis. This revealed striped domains that transformed to magnetic bubbles with an increasing field [7]. The bubbles appeared to be made up of concentric rings of varying winding directions, making them topologically equivalent to magnetic skyrmions $[7,8]$. The authors note that the bubbles may originate from the uniaxial magnetic anisotropy present in the material rather than the antisymmetric Dzyaloshinskii-Moriya interaction responsible 
(a)

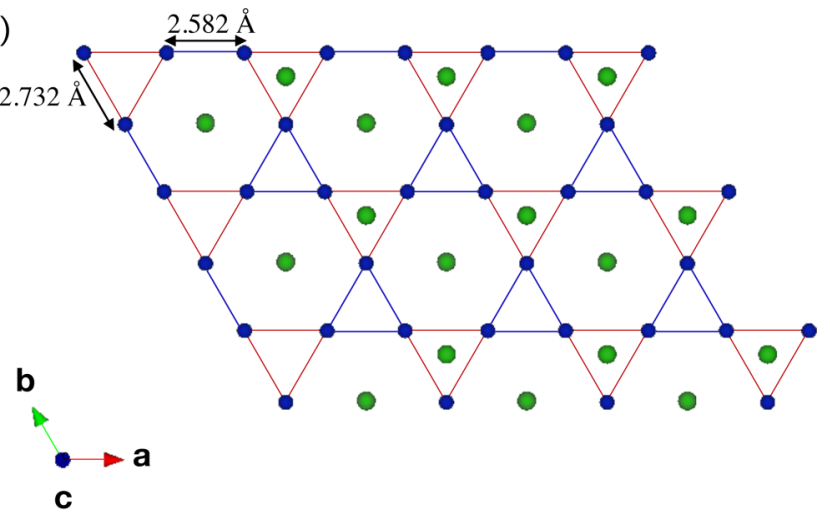

(b)

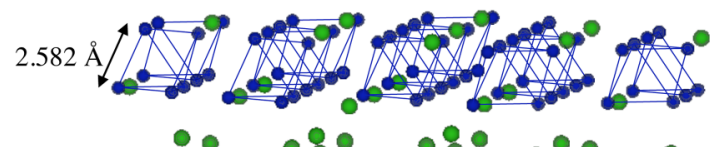

-
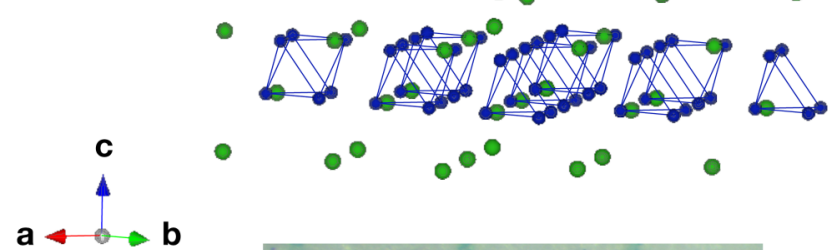

(c)

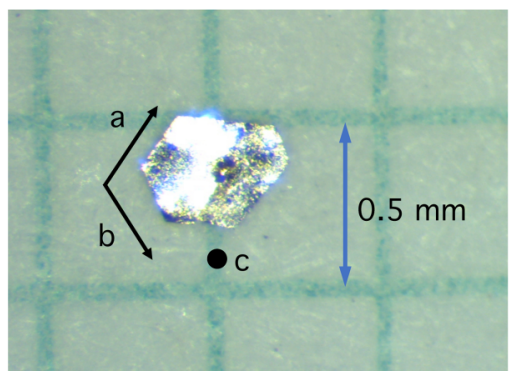

FIG. 1. (a) The crystal structure of $\mathrm{Fe}_{3} \mathrm{Sn}_{2}$ with the crystallographic axes shown as colored arrows. The blue $\mathrm{Fe}$ atoms form kagome planes made up of two sizes of equilateral triangles, 2.732 and $2.582 \AA$, shown in blue and red, respectively. (b) The kagome planes are bilayered where the $2.582-\AA \mathrm{Fe}-\mathrm{Fe}$ triangles form octahedra and the green Sn atoms sit within the kagome planes and between bilayers. (c) A typical hexagonal crystal of $\mathrm{Fe}_{3} \mathrm{Sn}_{2}$ showing the directions of the crystallographic axes along with a scale for comparison.

for the skyrmions in $\mathrm{MnSi}[7,8]$. The presence of skyrmionic bubbles is seen for fields up to $\approx 1.1 \mathrm{~T}$ and in the temperature range of 380-130 K [7]. For $\mu_{0} H>1.1 \mathrm{~T}$ all moments were seen to align with the $c$ axis. We now discuss our results that confirm that there is a topological magnetic Hall signal consistent with the presence of skyrmions.

\section{EXPERIMENT AND RESULTS}

Single crystals of $\mathrm{Fe}_{3} \mathrm{Sn}_{2}$ were grown by chemical vapor deposition from powdered material that had in turn been prepared from the elements. The powder of $\mathrm{Fe}_{3} \mathrm{Sn}_{2}$ was prepared from stoichiometric quantities of $\mathrm{Fe}$ and $\mathrm{Sn}$ that were ground together and pressed into a pellet that was placed into a silica ampoule and evacuated to $10^{-5} \mathrm{mb}$. Backfilling with argon was carried out to a pressure of $3.5 \mathrm{mb}$ to reduce Sn evaporation. The ampoule was then heated to $80^{\circ} \mathrm{C}$ at a ramp rate of $1{ }^{\circ} \mathrm{C} \min ^{-1}$ and left for 7 days. The reaction was then quenched by submersion in cold water. Single crystals were prepared by chemical vapor transport with $40 \mathrm{mg}$ of iodine as a carrier and $500 \mathrm{mg}$ of the prepared $\mathrm{Fe}_{3} \mathrm{Sn}_{2}$ powder loaded into a $16-\mathrm{cm}$-long silica ampoule. The ampoule was evacuated to a pressure of $10^{-6} \mathrm{mb}$ and sealed. After heating at a rate of $1{ }^{\circ} \mathrm{C} \mathrm{min}^{-1}$ in a two-zone furnace to temperatures of 650 and $720^{\circ} \mathrm{C}$, the reaction was left for 8 days and yielded crystals up to $6 \mathrm{~mm}$ in diameter. Typical single crystals are hexagonal or triangular in shape, with dimensions of $\approx 0.5 \times$ $0.5 \times 0.04 \mathrm{~mm}$, as shown in Fig. 1(c). X-ray Laue diffraction confirmed that the $c$ axis is perpendicular to the plane, while the $a$ and $b$ axes are parallel to the edges of the hexagon.

Magnetization measurements were carried out on a highquality single crystal $[R R R=28$; Fig. 3(a)] using a SQUID magnetometer (MPMS-XL7; Quantum Design, Inc.). The DC susceptibility, $M / H$, namely, the magnetization $M$ divided by $\mu_{0} H$, is shown in SI units as a function of the temperature from 300 to $2 \mathrm{~K}$ for $\mu_{0} H=0.05 \mathrm{~T} \|$ to the $c$ axis (blue) and $\mu_{0} H=0.01 \mathrm{~T} \|$ to the $a$ axis (black). Hysteresis curves were carried out at a constant temperature, following the $\mu_{0} H$ ramp profile of $0 \mathrm{~T} \rightarrow 4 \mathrm{~T} \rightarrow-4 \mathrm{~T} \rightarrow 0 \mathrm{~T}$. The $1 \mathrm{~T} \rightarrow$ $0 \mathrm{~T}$ and $4 \mathrm{~T} \rightarrow 0 \mathrm{~T}$ segments of the hysteresis curves for $\mu_{0} H \|$ to the $a$ axis and $c$ axis, respectively, expressed in Bohr magnetons per Fe are shown in Figs. 2(b), 2(d), and 2(e). At all measured temperatures and field directions no notable hysteresis is observed and the curves for increasing and decreasing fields are indistinguishable as shown in the full $\pm 4 \mathrm{~T}$ hysteresis curve in Fig. 2(c) at $120 \mathrm{~K}$ for $\mu_{0} H \|$ to the $c$ axis.

Gold $25-\mu \mathrm{m}$ contacts were added along and across the $a-b$ plane of the same sample used in the magnetization study in a Hall bar geometry, perpendicular to the $c$ axis, using Dupont 4929 silver epoxy. A current of $200 \mu$ A was applied in the $a-b$ plane at a frequency of $37 \mathrm{~Hz}$ and the voltage signal measured using a transformer and lock-in amplifier. The zero-field resistivity, $\rho_{x x}(0)$, versus temperature is shown in Fig. 3(a), demonstrating metallic like behavior and a sample $\mathrm{RRR}=28$. The magnetoresistance, $\rho_{x x}(H)$, and Hall resistivity, $\rho_{x y}$, were measured concurrently in the $a-b$ plane at a constant temperature with $\mu_{0} H \|$ to the $c$ axis following a $\pm 4 \mathrm{~T}$ ramp profile identical to that used in the magnetization study. The $\rho_{x x}(H)$ and $\rho_{x y}$ curves were symmetrized and antisymmetrized, respectively, for positive and negative fields to correct for any contact misalignment. The measured curves of the change in resistivity relative to the zero-field value, i.e., $\left(\rho_{x x}(H)-\rho_{x x}(0)\right) / \rho_{x x}(0)$, versus $\mu_{0} H$ up to $4 \mathrm{~T}$ are shown in Figs. 3(b) and 3(c). The corresponding $\rho_{x y}$ curves are shown in Fig. 4.

In a collinear ferromagnet $\rho_{x y}$ is made up of (i) the ordinary Hall effect, $\rho_{x y}^{n}$, dependent on $n$, the free carrier concentration, and (ii) the AHE, $\rho_{x y}^{\mathrm{AHE}}$, dependent on the sample magnetization, $M$, to give

$$
\rho_{x y}=\rho_{x y}^{n}+\rho_{x y}^{\mathrm{AHE}}=-\frac{\mu_{0} H}{n e}+R_{s} M,
$$

where $e$ is the elementary charge constant and $R_{s}$ is the anomalous Hall coefficient. The first microscopic description of the AHE by Karplus and Luttinger [16], known as the 

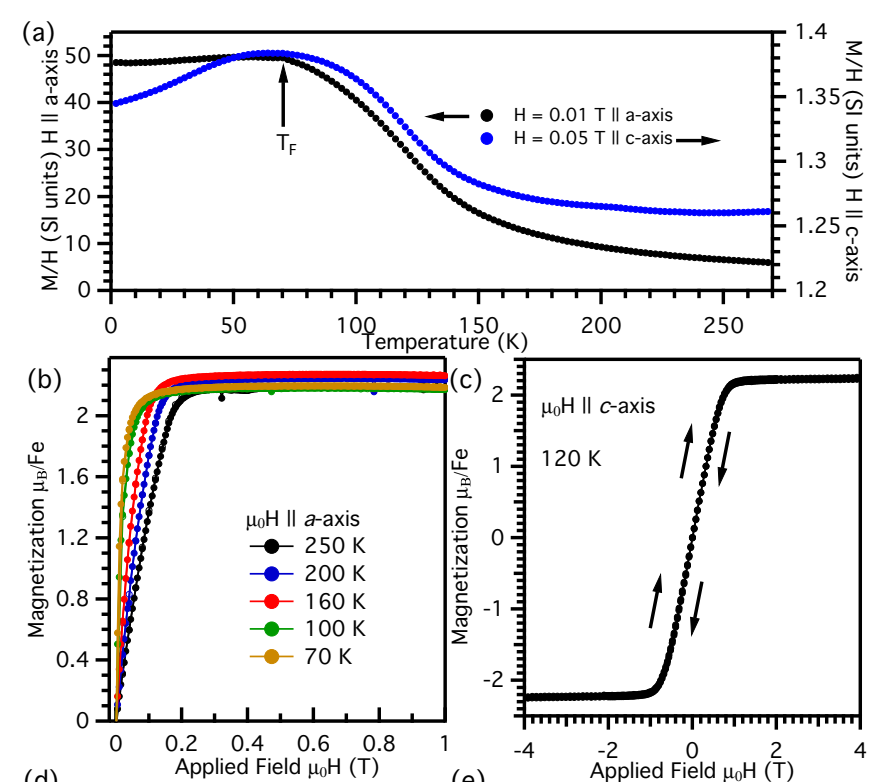

(d)
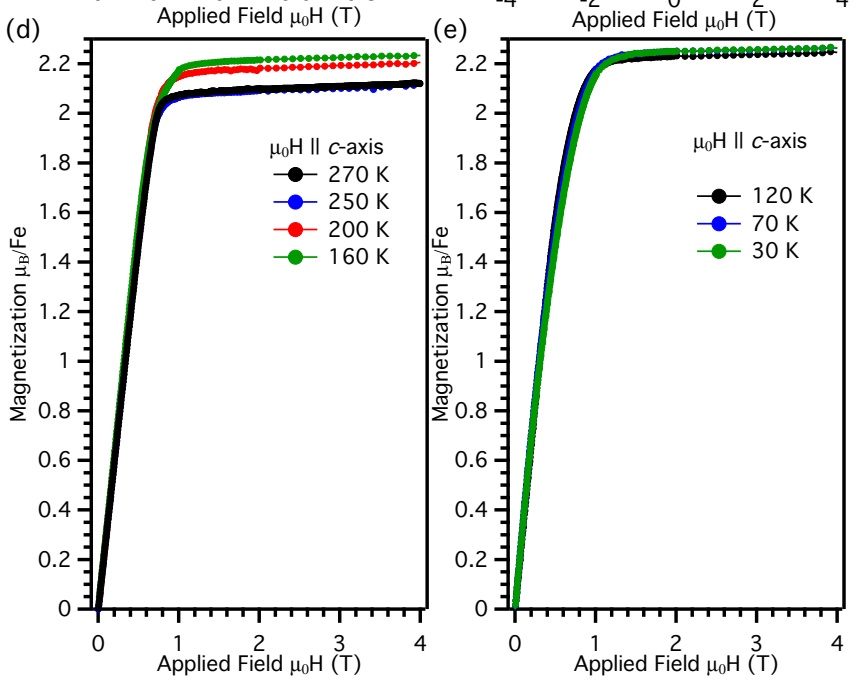

FIG. 2. (a) Curves of magnetic DC susceptibility $(M / H)$ versus temperature, for $\mu_{0} H=0.01$ and $0.05 \mathrm{~T}$ applied parallel to the $a$ axis (left axis) and $c$ axis (right axis), respectively. The maximum in $(M / H)$ attributed to $T_{F}=70 \mathrm{~K}$ is indicated. (b) The $1 \mathrm{~T} \rightarrow 0 \mathrm{~T}$ segment of hysteresis curves for $\mu_{0} H$ parallel to the $a$ axis at a series of temperatures. (c) The full magnetic hysteresis curve at $120 \mathrm{~K}$ between $\pm 4 \mathrm{~T}$, for $\mu_{0} H$ parallel to the $c$ axis. No notable hysteresis is observed. (d, e) The $4 \mathrm{~T} \rightarrow 0 \mathrm{~T}$ segment of hysteresis curves at a series of temperatures for $\mu_{0} H$ parallel to the $c$ axis.

intrinsic AHE, depended solely on the topology of the material's band structure including the spin-orbit interaction. In modern notation, the intrinsic effect is expressed in terms of the Berry curvature given by

$$
R_{s}=\frac{e^{2}}{\hbar}\langle\Omega\rangle \rho_{x x}^{2}=S_{H} \rho_{x x}^{2}
$$

Here $\langle\Omega\rangle$ is the total weighted-average Berry curvature for the band structure and $S_{H}$ is a temperature-independent scaling factor. A nonzero $\langle\Omega\rangle$ causes carriers to experience an extra anomalous Hall velocity perpendicular to the applied electric field [17].
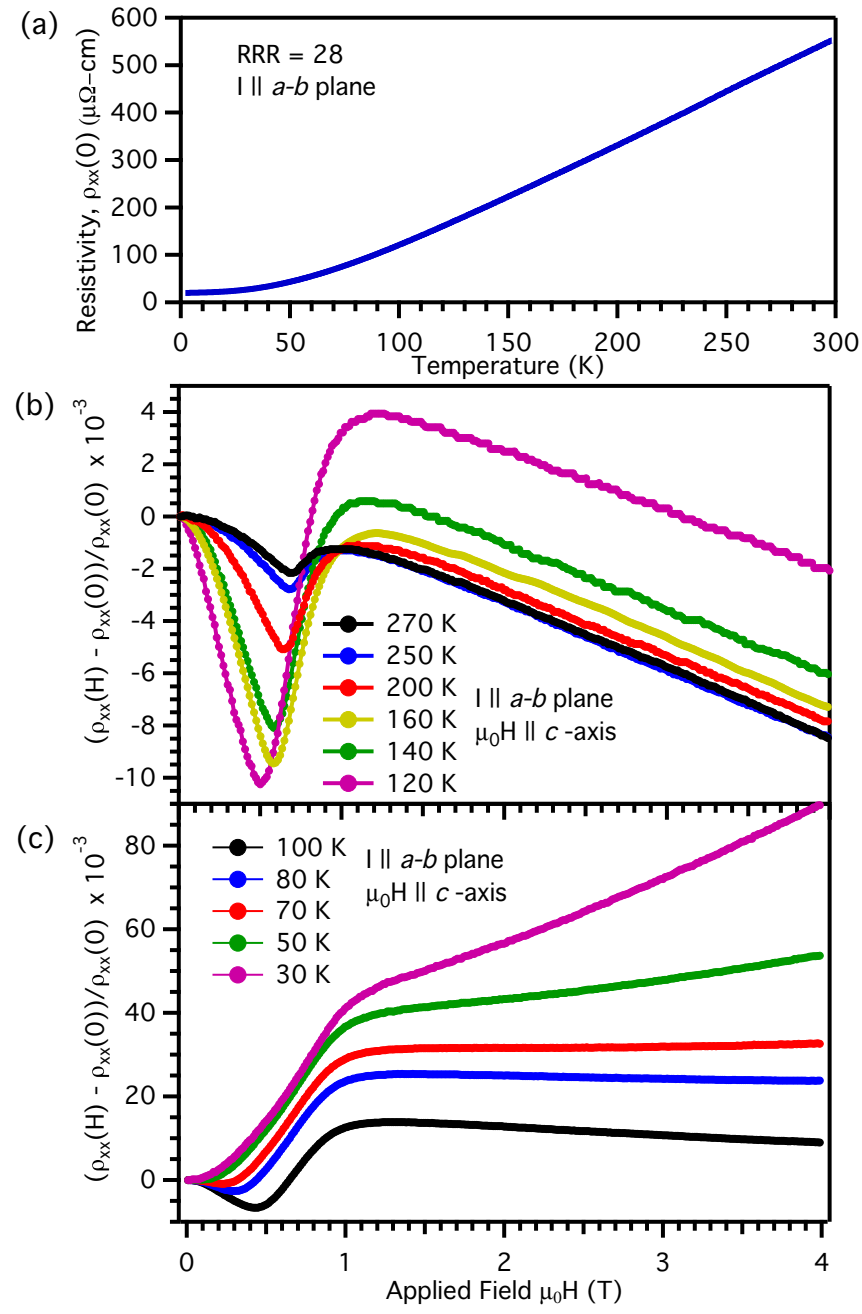

FIG. 3. (a) The zero-field resistivity, $\rho_{x x}(0)$, of $\mathrm{Fe}_{2} \mathrm{Sn}_{3}$ measured in the $a-b$ plane of the crystal. ( $\mathrm{b}, \mathrm{c})$ Curves of the change in resistivity relative to the zero-field value versus $\mu_{0} H$ for a range of temperatures between 270 and $30 \mathrm{~K}$.

There are also two extrinsic contributions to $R_{s}$, denoted skew and side-jump scattering. Here the spin-orbit interaction causes asymmetrical scattering of conduction electron wavepackets from impurities $[18,19]$. This asymmetry leads to a Hall voltage that scales linearly with the longitudinal resistivity, $\propto \rho_{x x}$ for skew scattering and quadratically $\propto \rho_{x x}^{2}$ for side-jump scattering. Hence it is difficult experimentally to distinguish between intrinsic and side-jump contributions. In transition-metal ferromagnets such as $b c c \mathrm{Fe}$, which possess nonsimplistic band structures and large Berry curvatures, the intrinsic AHE is thought to far outweigh the side-jump mechanism [20].

Fits according to Eqs. (1) and (2) to determine $\rho_{x y}^{n}$ and $\rho_{x y}^{\mathrm{AHE}}$ contributions to $\rho_{x y}$ in $\mathrm{Fe}_{3} \mathrm{Sn}_{2}$ using the measured curves of $M$ and $\rho_{x x}(H)$ and taking into account demagnetizing fields were carried out as follows. The magnetization curves show that moments become aligned with $\mu_{0} H \|$ to the $c$ axis above a saturation field $H \geqslant H_{S}(\approx 1 \mathrm{~T})$. The slope of the hysteresis curves below $H_{s}$ in Figs. 2(d) and 2(e) are almost temperature independent and determined by the demagnetization factor, $N$. 


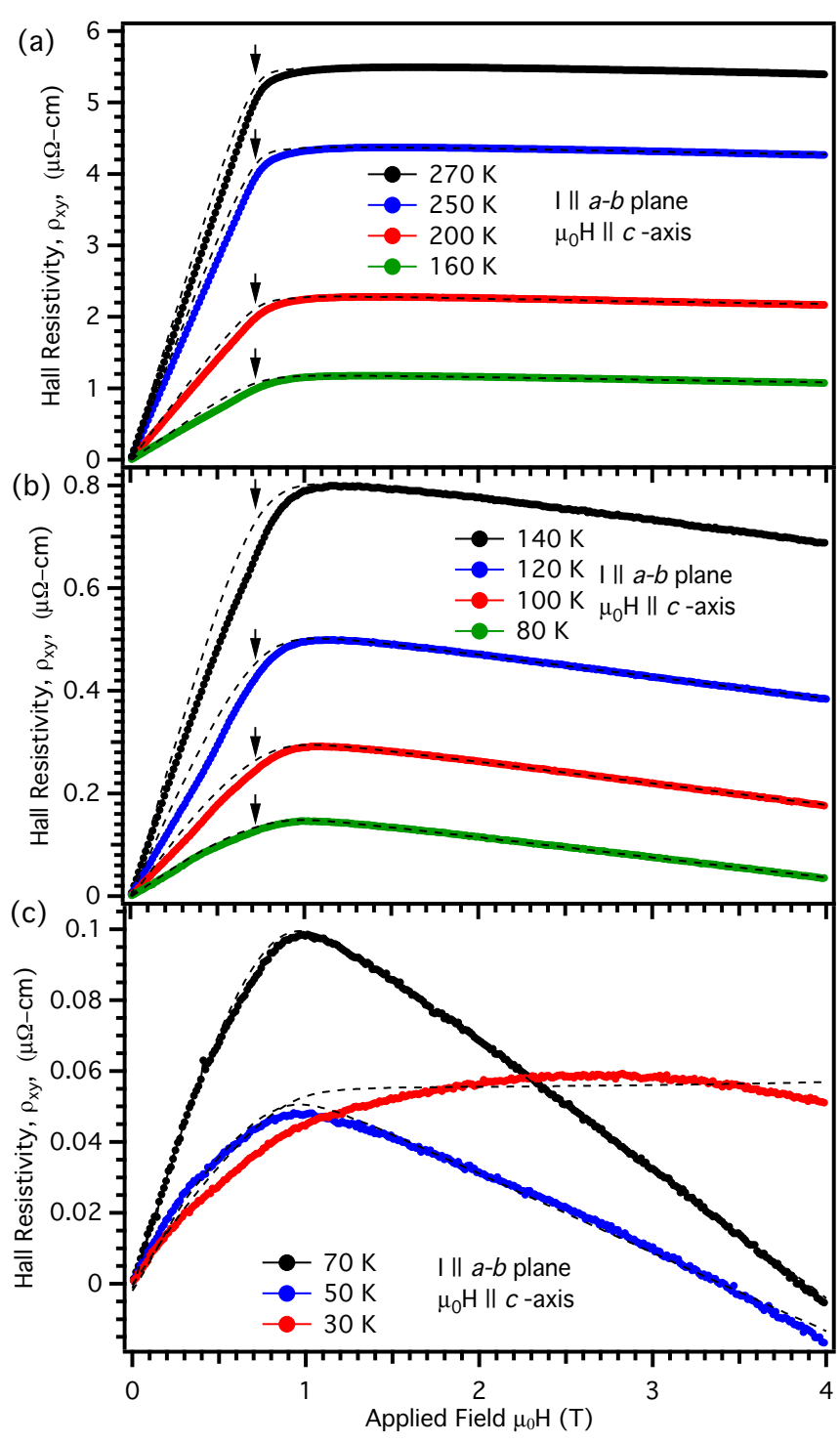

FIG. 4. (a-c) Curves of Hall resistivity, $\rho_{x y}$, versus $\mu_{0} H$, between 270 and $30 \mathrm{~K}$. Dashed black lines are calculated fits based on Eqs. (1) and (2) discussed in the text. Black arrows indicate the region where an extra contribution to the Hall resistivity, $\Delta \rho_{x y}$, exists.

For our sample geometry the expected demagnetizing factor is calculated to be $N=0.796$ [21]. An experimental upper limit for $N$ can be obtained from the maximum slope of $d M / d H$ to give $N<0.721$ from the data at $270 \mathrm{~K}$. The value of 0.721 was used to estimate the effective field $\mu_{0} H_{\text {eff }}$ correcting for the demagnetizing field,

$$
\mu_{0} H_{\mathrm{eff}}=\mu_{0} H-N M .
$$

Since the same sample and field history was used in the magnetization, magnetoresistance, and Hall effect measurements, $N$ and hence $\mu_{0} H_{\text {eff }}$ have the same values for all these quantities. Below the saturation field, $\mu_{0} H_{\text {eff }}$ is close to 0 and the Hall resistivity is dominated by terms depending only on the value of $M$, principally the anomalous Hall term. Above the saturation field, a straightforward fit to $\rho_{x y}$ vs $\mu_{0} H_{\text {eff }}$ based on Eqs. (1) and (2) is made. This is independent of any dependence of $N$ on the value of $M$, since $M$ is the same in
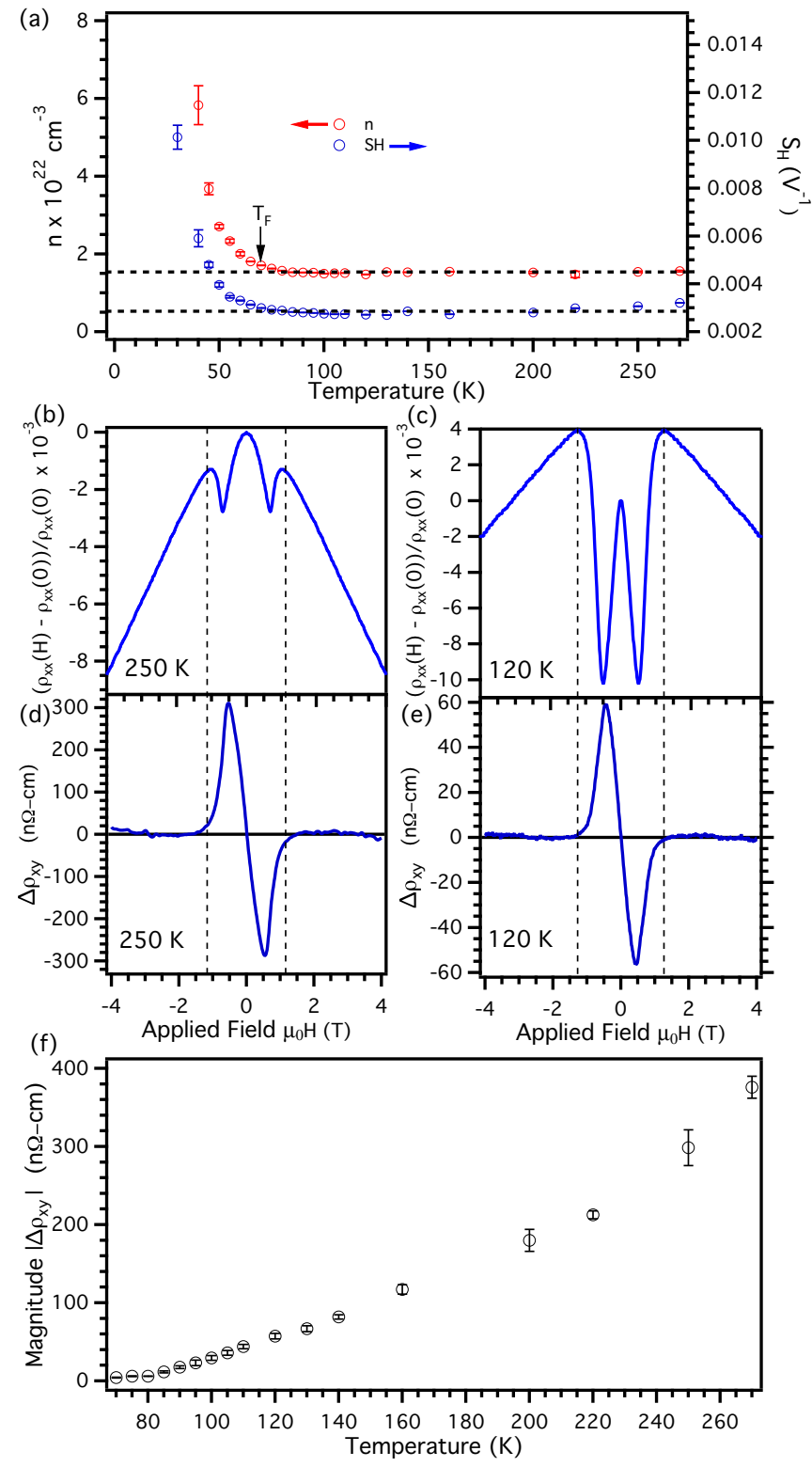

FIG. 5. (a) Values of $n$ (left axis) and $S_{H}$ (right axis) from fits to the Hall resistivity as a function of the temperature. Dashed horizontal lines are guides for the eye to demonstrate the temperature independence of $n$ and $S_{H}$ above $T_{F}$. (b, c) Curves of the change in resistivity relative to the zero-field value versus $\mu_{0} H$ for 250 and $120 \mathrm{~K}$, respectively. (d, e) The corresponding $\Delta \rho_{x y}$ curves. Dashed vertical lines are guides for the eye showing that $\Delta \rho_{x y}$ exists over the same $\mu_{0} H$ range as the anomaly seen in magnetoresistance. (f) The magnitude of the peak maximum $\left|\Delta \rho_{x y}\right|$ averaged for both positive and negative fields as a function of the temperature from $270 \mathrm{~K}$ to $T_{F}$.

both measurements. If the scaling factor $S_{H}$ is considered to be field independent, fits to $\rho_{x y}$ given by Eqs. (1) and (2) can then be calculated over the full field range of $\mu_{0} H_{\text {eff }}$ and are shown by the dashed lines in Fig. 4. The values of $n$ and $S_{H}$ determined from the fits are shown as a function of the temperature in Fig. 5(a). The differences between $\rho_{x y}$ and the calculated value are discussed in more detail below. 


\section{DISCUSSION}

We first consider the transition to the spin-glass state at $T_{F}$. Previous reports saw a transition at $T_{F} \approx 80 \mathrm{~K}$, where $T_{F}$ was determined by a maximum in magnetic susceptibility [6]. Here a similar maximum in the DC magnetic susceptibility, $M / H$, along both the $a$ and the $c$ axis directions is indicated by the arrow in Fig. 2(a). We define $T_{F}$ as the temperature at which $M / H$ reaches the maximum, giving $T_{F}=70 \pm 2.5 \mathrm{~K}$. Additionally the curves of magnetoresistance in Figs. 3(b) and 3(c) show a large negative anomaly for $\mu_{0} H<1 \mathrm{~T}$ that becomes suppressed below $70 \mathrm{~K}$ and the calculated values of $n$ and $S_{H}$ from the Hall effect fitting strongly increase below $70 \mathrm{~K}$ [Fig. 5(a)]. The difference in $T_{F}$ from previous reports may be due to their polycrystalline samples being of a lower quality, extra demagnetizing effects from the random grain orientations, or the presence of extra strains/dislocations.

Above $T_{F}$ excellent agreement is seen between $\rho_{x y}$ and the fit to Eqs. (1) and (2) for $\mu_{0} H>H_{s}(\approx 1 \mathrm{~T})$. However, below $T_{F}$ the fits become progressively worse upon cooling, for example, the curves at 50 and $30 \mathrm{~K}$ in Fig. 4(c). The poor fitting may be due to an unconventional AHE or a change in Fermi surface in the spin-glass state.

The remainder of this paper focuses on temperatures above $T_{F}$. At fields below saturation $\left(\mu_{0} H<H_{s}\right)$ a significant deviation between the fit and the data, indicated by the black arrows in Figs. 4(a) and 4(b), exists. This region in $\mathrm{Fe}_{3} \mathrm{Sn}_{2}$ is expected to contain noncollinear moments possessing both $a-b$ plane and $c$ axis components, which here we label the $\mathrm{R}$ state. The difference between $\rho_{x y}$ and calculated fits is denoted $\Delta \rho_{x y}$. While extrinsic responses are expected to become more prominent as the temperature is lowered due to longer relaxation times, including a skew scattering term, $a \rho_{x x}(H)$, where $a$ is a scaling constant, does not reduce $\Delta \rho_{x y}$. Fermi surface measurements and band structure calculations for $\mathrm{Fe}_{3} \mathrm{Sn}_{2}$ show a multiband system with a complex Fermi surface and large Berry curvature [22-24]. Along with the temperature independence of $S_{H}$ and $n$ above $T_{F}$ in Fig. 5(a), it can be concluded that the AHE is intrinsic in accordance with previous reports [10,25]. Although side-jump scattering also scales with $\rho_{x x}^{2}$, for such a multiband system the intrinsic effect is expected to far outweigh any side-jump contribution.

Curves of $\Delta \rho_{x y}$ are shown in units of $\mathrm{n} \Omega$-cm versus $\mu_{0} H$ in Figs. 5(d) and 5(e) for 250 and $120 \mathrm{~K}$, respectively, while the magnitude of the peak maximum $\left|\Delta \rho_{x y}\right|$ averaged for both positive and negative fields is plotted as a function of the temperature in Fig. 5(f). The field range over which $\Delta \rho_{x y}$ exists corresponds directly to that of the negative anomaly in magnetoresistance, shown in Figs. 5(b) and 5(c). Such negative magnetoresistance anomalies in noncollinear magnetic materials may be associated with the presence of magnetic fluctuations, which increase resistivity, being suppressed as moments become aligned with $\mu_{0} H$ [26].

The $\Delta \rho_{x y}$ present in the $\mathrm{R}$ state of $\mathrm{Fe}_{3} \mathrm{Sn}_{2}$ may represent a THE like that in the skyrmion lattice of MnSi [4]. While the magnitude of $\Delta \rho_{x y}(\approx 180 \mathrm{n} \Omega-\mathrm{cm}$ at $200 \mathrm{~K})$ is significantly larger and opposite in sign to that seen for MnSi [4], it is comparable to the THE of MnGe, where the period of the chirality is much shorter and a large negative THE exists below $60 \mathrm{~K}$ [27]. As discussed in Sec. I Lorentz transmission electron

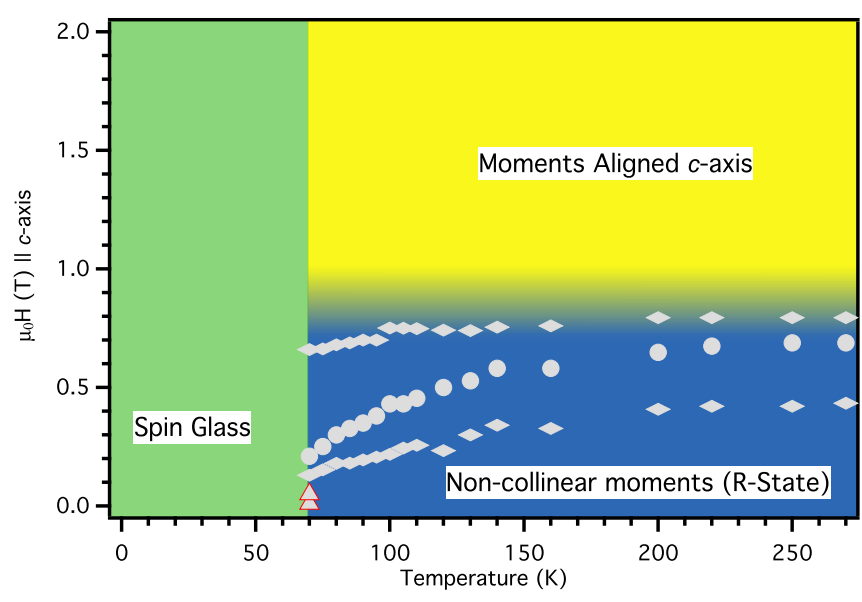

FIG. 6. The $\mu_{0} H-T$ phase diagram of $\mathrm{Fe}_{3} \mathrm{Sn}_{2}$ with $\mu_{0} H$ parallel to the $c$ axis. The green region represents the spin-glass phase below $T_{F}=70 \mathrm{~K}$ determined from the magnetic susceptibility, shown as gray triangles outlined in red. Gray circle and diamond markers are derived from the anomaly in magnetoresistance, as described in the text. The blue area depicts the R-state region of noncollinear moments, while the yellow area represents moment saturation with $\mu_{0} H$ and the shaded blue/yellow area represents the crossover region.

microscopy measurements detected spin-textured magnetic bubbles topologically equivalent to skyrmions in $\mathrm{Fe}_{2} \mathrm{Sn}_{3}$ from 380 to $130 \mathrm{~K}$ up to $1.1 \mathrm{~T}$, coinciding well with the temperature and field range of the largest $\Delta \rho_{x y}$ effect seen here. Upon cooling towards $T_{F}$ the density of the spin-textured magnetic bubbles was observed to decrease $[7,8]$. The magnetization curves in Fig. 2(b) show a decrease in the saturation field approaching $T_{F}$ indicative of an increase in the $a-b$ moment. These observations may provide an explanation for the decreasing magnitude of $\Delta \rho_{x y}$ upon cooling and suggest the need for a significant $c$ axis component of noncollinear moments for $\Delta \rho_{x y}$ to exist. Similarly since the R state contains moments with both $a-b$ plane and $c$-axis components, a decrease in magnitude is expected for temperatures higher than those accessible here that contain a smaller $a-b$ moment component. Then $\left|\Delta \rho_{x y}\right|$ will follow a dome-shaped dependence to higher temperatures. A THE of a similar magnitude and temperature dependence has recently been reported in $\mathrm{Mn}_{1.5} \mathrm{PtSn}$ [28].

The magnetic phase diagram of $\mathrm{Fe}_{3} \mathrm{Sn}_{2}$ for $\mu_{0} H \|$ to the $c$ axis is shown in Fig. 6. The transition to the spin-glass phase is shown as the green region, where the red-outlined gray triangles indicate the $T_{F}$ determined from the magnetic susceptibility. The vertical line defining the field dependence of $T_{F}$ is inferred from the behavior of the Hall and magnetoresistance signals being clearly different to the left and right of this line as shown in Figs. 3(c) and 4(c). A vertical line is also reported in the literature by Hou et al. [7]. The gray circle and diamond markers represent the minimum and half-maxima of the anomaly in magnetoresistance, respectively. The blue and yellow regions indicate the $\mathrm{R}$ and fully aligned states, respectively, while the shaded blue/yellow area represents the crossover region. The crossover is defined as the region between the second half-maximum in the anomaly of magnetoresistance and the saturation field $H_{s}$. 
Other contributions to $\Delta \rho_{x y}$ not due to a THE should also be considered. The lack of any measurable hysteresis and high value of the RRR suggest that the existence of $\Delta \rho_{x y}$ is not due to magnetic domains or disorder. As already discussed $\Delta \rho_{x y}$ is not due to skew scattering and the sidejump mechanism is expected to be far outweighed by the intrinsic effect. The fitting used here assumes a linear ordinary Hall effect response without consideration of different carrier types or multiband effects. However, for fields above moment saturation where the ordinary Hall effect dominates, the linear response fits well with only electron carriers, as shown by the small error bars in $n$ above $T_{F}$. In addition to this the temperature independence of $n$ and $S_{H}$ above $T_{F}$ suggests that any changes in Fermi surface topology here are unlikely.

\section{CONCLUSIONS}

In summary we have detected an extra contribution in the Hall resistivity of $\mathrm{Fe}_{3} \mathrm{Sn}_{2}$ above $T_{F}$ that may be due to the topological nature of spin textures present in the noncollinear moment $\mathrm{R}$ state below moment saturation. This is the first report of such an anomaly in $\mathrm{Fe}_{3} \mathrm{Sn}_{2}$ and the first of its kind in a kagome structured material. The feature in Hall resistivity is complimented by a negative anomaly in magnetoresistance.

\section{ACKNOWLEDGMENTS}

Support from the Engineering and Physical Sciences Research Council, EP/P013686/1 and EP/J00099X/1 (C.D.O’N.) and (A.D.H.), is acknowledged.
[1] P. Bruno, V. K. Dugaev, and M. Taillefumier, Phys. Rev. Lett. 93, 096806 (2004).

[2] S. Mühlbauer, B. Binz, F. Jonietz, C. Pfleiderer, A. Rosch, A. Neubauer, R. Georgii, and P. Böni, Science 323, 915 (2009).

[3] Y. Taguchi, Y. Oohara, H. Yoshizawa, N. Nagaosa, and Y. Tokura, Science 291, 2573 (2001).

[4] A. Neubauer, C. Pfleiderer, B. Binz, A. Rosch, R. Ritz, P. G. Niklowitz, and P. Böni, Phys. Rev. Lett. 102, 186602 (2009).

[5] R. Ritz, M. Halder, C. Franz, A. Bauer, M. Wagner, R. Bamler, A. Rosch, and C. Pfleiderer, Phys. Rev. B 87, 134424 (2013).

[6] L. A. Fenner, A. A. Dee, and A. S. Wills, J. Phys.: Condens. Matter 21, 452202 (2009).

[7] Z. P. Hou, W. J. Ren, B. Ding, G. Z. Xu, Y. Wang, B. Yang, Q. Zhang, Y. Zhang, E. K. Liu, F. Xu, W. H. Wang, G. H. Wu, X. X. Zhang, B. G. Shen, and Z. D. Zhang, Adv. Mater. 29, 1701144 (2017)

[8] J.-X. Yin, S. S. Zhang, H. Li, K. Jiang, G. Chang, B. Zhang, B. Lian, C. Xiang, I. Belopolski, H. Zheng et al., Nature 562, 91 (2018)

[9] B. Malaman and B. Roques, Acta Crystallogr. B32, 1348 (1976).

[10] Q. Wang, S. Sun, X. Zhang, F. Pang, and H. Lei, Phys. Rev. B 94, 075135 (2016).

[11] G. Trumpy, E. Both, C. Djéga-Mariadassou, and P. Lecocq, Phys. Rev. B 2, 3477 (1970).

[12] G. L. Caer, B. Malaman, and B. Roques, J. Phys. F: Metal Phys. 8, 323 (1978)

[13] G. L. Caer, B. Malaman, L. Haggstrom, and T. Ericsson, J. Phys. F 9, 1905 (1979).

[14] B. Malaman, D. Fruchart, and G. L. Caer, J. Phys. F 8, 2389 (1978).
[15] M. Pereiro, D. Yudin, J. Chico, C. Etz, O. Eriksson, and A. Bergman, Nat. Commun. 5, 4815 (2014).

[16] R. Karplus and J. M. Luttinger, Phys. Rev. 95, 1154 (1954).

[17] M. Lee, Y. Onose, Y. Tokura, and N. P. Ong, Phys. Rev. B 75, 172403 (2007).

[18] J. Smit, Physica 24, 39 (1958).

[19] L. Berger, Phys. Rev. B 2, 4559 (1970).

[20] Y. Yao, L. Kleinman, A. H. MacDonald, J. Sinova, T. Jungwirth, D.-S. Wang, E. Wang, and Q. Niu, Phys. Rev. Lett. 92, 037204 (2004).

[21] R. B. Goldfarb and J. V. Minervini, Rev. Sci. Instrum. 55, 761 (1984).

[22] L. Ye, M. Kang, J. Liu, F. von Cube, C. R. Wicker, T. Suzuki, C. Jozwiak, A. Bostwick, E. Rotenberg, D. C. Bell et al., Nature 555, 638 (2018).

[23] L. Ye, M. K. Chan, R. D. McDonald, D. Graf, M. Kang, J. Liu, T. Suzuki, R. Comin, L. Fu, and J. G. Checkelsky, arXiv:1809.11159.

[24] Z. Lin, J.-H. Choi, Q. Zhang, W. Qin, S. Yi, P. Wang, L. Li, Y. Wang, H. Zhang, Z. Sun et al., Phys. Rev. Lett. 121, 096401 (2018).

[25] T. Kida, L. A. Fenner, A. A. Dee, I. Terasaki, M. Hagiwara, and A. S. Wills, J. Phys.: Condens. Matter 23, 112205 (2011).

[26] G. Abdul-Jabbar, D. A. Sokolov, C. D. O’Neill, C. Stock, D. Wermeille, F. Demmel, F. Krüger, A. G. Green, F. LévyBertrand, B. Grenier et al., Nat. Phys. 11, 321 (2015).

[27] N. Kanazawa, Y. Onose, T. Arima, D. Okuyama, K. Ohoyama, S. Wakimoto, K. Kakurai, S. Ishiwata, and Y. Tokura, Phys. Rev. Lett. 106, 156603 (2011).

[28] P. Swekis, A. Markou, D. Kriegner, J. Gayles, R. Schlitz, W. Schnelle, S. T. B. Goennenwein, and C. Felser, Phys. Rev. Mater. 3, 013001 (2019). 